\title{
Outcome of patients with lung cancer and severe psychiatric disorder admitted to a medical psychiatric unit
}

\author{
TOMOHIRO TAKEHARA ${ }^{1,2}$, TETSUO TANI ${ }^{2}$, HIROYUKI TAKIUE ${ }^{3}$, RYO TAKESHITA $^{2}$, \\ ARIFUMI IWAMARU ${ }^{4}$, TATSUYA YAMAMOTO ${ }^{4}$ and HIDEFUMI $\mathrm{KOH}^{2}$ \\ ${ }^{1}$ Division of Pulmonary Medicine, Department of Medicine, Keio University School of Medicine; \\ ${ }^{2}$ Division of Pulmonary Medicine, Department of Internal Medicine, Federation of National Public Service Personnel \\ Mutual Aid Associations, Tachikawa Hospital; Departments of ${ }^{3}$ Neuropsychiatry and ${ }^{4}$ Surgery, Federation of \\ National Public Service Personnel Mutual Aid Associations, Tachikawa Hospital, Tokyo 190-8531, Japan
}

Received February 18, 2019; Accepted September 19, 2019

DOI: $10.3892 / \mathrm{mco} .2020 .1974$

\begin{abstract}
The purpose of the present study was to evaluate the clinical profiles and treatment outcomes of patients with lung cancer admitted to the Medical Psychiatric Unit (MPU), which is built for patients with physical and severe psychiatric disorders. All medical records of patients with lung cancer admitted to the MPU of Tachikawa hospital were reviewed. The clinical outcomes of these patients were retrospectively evaluated between January 2010 and December 2016. A total of 24 patients in the MPU were histologically or cytologically diagnosed with primary lung cancer. Of these, 20 patients had schizophrenia, and 4 patients had a mood disorder. There were 15 patients who were diagnosed using bronchoscopy. The histology indicated adenocarcinoma, squamous cell carcinoma and non-small-cell lung cancer-not otherwise specified were in 11,8 , and 1 patient, respectively, while small-cell lung cancer was indicated in 4 patients. Surgery, chemoradiotherapy, radiotherapy, chemotherapy was performed in 13, 4, 2, 1 and 4 patients, respectively. The median survival time was 76.7 months for patients who underwent surgery, while it was 14.4 months for those who underwent chemoradiotherapy. In the MPU, patients with lung cancer and severe psychiatric disorders could be safely diagnosed, and patients with early-stage lung cancer exhibited long-term survival.
\end{abstract}

Correspondence to: Dr Tetsuo Tani, Division of Pulmonary Medicine, Department of Internal Medicine, Federation of National Public Service Personnel Mutual Aid Associations, Tachikawa Hospital, 4-2-22 Nishikicho, Tachikawa, Tokyo 190-8531, Japan E-mail: tetsuo19840324@yahoo.co.jp

Key words: lung cancer, psychiatric disorder, schizophrenia, mood disorder, medical psychiatric unit

\section{Introduction}

Lung cancer is the leading cause of cancer-related mortality worldwide (1). The incidence of heavy smoking is higher in those with psychiatric disorders than that in the general population $(2,3)$, and the incidence of lung cancer in patients with schizophrenia is significantly higher than that in the general population (4). In addition, the mortality rate in patients with lung cancer with schizophrenia is higher than that in the general population (5). However, the cause for the increased lung cancer mortality rate in patients with schizophrenia is poorly understood.

In general, it is challenging to determine the appropriate diagnostic and treatment modality for patients with lung cancer with psychiatric disorders due to difficulties in obtaining their cooperation and consent for treatment. It is also estimated that many patients with psychiatric conditions are not properly diagnosed or treated due to their deteriorated mental status during examination and treatment. Patients with lung cancer who have schizophrenia have acceptable postoperative outcomes (6), but studies on the outcome of patients with lung cancer with severe psychiatric disorders who need to be hospitalized are limited.

Our hospital in Japan has a Medical Psychiatric Unit (MPU) where patients with psychiatric conditions who require admission to a psychiatric facility are admitted for examination and treatment of physical conditions (7). In the MPU, patients are co-managed by both psychiatrists and physicians. This study aimed to evaluate the clinical profiles and treatment results of patients with lung cancer with severe psychiatric disorders who were admitted to the MPU.

\section{Patients and methods}

We retrospectively reviewed the clinical profiles, diagnostic methods, and treatment courses for patients who were admitted to the MPU from January 2010 to December 2016 and diagnosed with primary lung cancer. All patients had been previously admitted to a psychiatric hospital to receive treatment for severe psychiatric disorders diagnosed by the ICD-10 classification. The patients were referred to our 
hospital for chest radiograph abnormalities, fever, difficulty of breathing, and other reasons. All included patients were histologically diagnosed with primary lung cancer. Staging was according to the 8th edition of the Union for International Cancer Control TNM classification. Kaplan-Meier curves were used to assess overall survival.

The patients' mental status was assessed daily by their psychiatrists during hospitalization, and treatments for their psychiatric disorders were adjusted as necessary. Written informed consent was obtained from patients and/or their legal guardian or key family member.

This study was conducted in accordance with the 1964 Declaration of Helsinki, as revised in 2013, and was approved by the Ethical review board of Tachikawa Hospital (Tokyo, Japan).

\section{Results}

A total of 24 patients were included in the analysis. The median patient age was 64 years (range, 53-75 years). The patients were referred to our hospital for chest radiograph abnormalities $(n=17)$, fever $(n=3)$, difficulty of breathing $(n=2)$, and other reasons $(n=2)$. In total, 20 and 4 were diagnosed with schizophrenia and mood disorders, respectively. All patients had severe psychiatric symptoms requiring hospitalization in a psychiatric facility for a long period of time even though their psychotropic medications were adjusted. In patients with schizophrenia, 5 patients took first-generation antipsychotics, 14 patients took second- generation antipsychotics, and one patient took no medications. The histological type of cancer was adenocarcinoma in 11 patients, squamous cell carcinoma in 8 patients, non-small cell lung carcinoma-not otherwise specified in 1 patient, and small cell lung cancer in 4 patients. The baseline patient characteristics are shown in Table I. Fifteen patients were diagnosed via bronchoscopy, while 6, 2, and 1 patient was diagnosed via CT-guided percutaneous lung biopsy, surgical biopsy, and sputum cytology, respectively. All patients had no family history on lung cancer. In addition, there were $10,2,6,3$, and 3 patients who had clinical stage I, II, IIIA, IIIB, and IV disease, respectively. Regarding comorbidities, 3 patients had interstitial pneumonia or chronic obstructive pulmonary disease, and 5 patients had diabetes mellitus. The oxygenation level of all patients as assessed according to arterial blood gas was normal. Echocardiography showed that all patients were eligible for surgery.

Regarding the type of treatment, 13, 4, 2, 1, and 4 patients underwent surgery, chemoradiotherapy, radiotherapy, chemotherapy and received best supportive care (BSC), respectively (Table II). Two patients with good performance status (case 10 and 21) did not undergo treatment and instead chose BSC based on an individual or family decision. Among the 13 patients who underwent surgery, lobectomy was performed in 11, while segmentectomy of the left upper division and wedge resection was performed in 1 patient each. Of the 4 patients with small-cell lung cancer, 1 underwent chemoradiotherapy, 1 patient underwent chemotherapy, and 2 patients received BSC.

Among those who underwent surgery, 5 cases of postoperative complications were recorded: Aspiration pneumonia
Table I. Patient characteristics.

\begin{tabular}{lc}
\hline Patient characteristics & $\begin{array}{c}\text { No. of } \\
\text { patients }\end{array}$ \\
\hline Total enrolled & 24 \\
Age (years) & \\
Median (range) & $64(53-75)$ \\
Sex & \\
Male & 14 \\
Female & 10
\end{tabular}

Type of psychiatric disorder

Schizophrenia 20

Mood disorder 4

ECOG performance status (pre-treatment)

0

1

2

3

3

2

2

4

0

Histology

Adenocarcinoma $\quad 11$

Squamous cell carcinoma $\quad 8$

NSCLC-NOS 1

Small-cell lung cancer 4

Clinical stage (TNM classification, 8th edition)

I

II

IIIA/IIIB $\quad 6 / 3$

IV 3

Comorbidity

Diabetes mellitus $\quad 5$

Interstitial pneumonia 3

Chronic obstructive pulmonary disease $\quad 3$

Diagnostic method

Bronchoscopy 15

CT-guided percutaneous lung biopsy $\quad 6$

Surgical biopsy $\quad 2$

Cytology 1

ECOG, Eastern Cooperative Oncology Group; NSCLC-NOS, non-small cell lung cancer-not otherwise specified; TNM, tumor node metastasis.

$(n=1)$, fistulous empyema $(n=1)$, and psychosis $(n=3)$ (Table III). In addition, grade 3/4 adverse events (Common Terminology Criteria for Adverse Events, version 4) occurred in 4 cases of chemoradiotherapy: Pneumonitis, 2 cases (Grade 3); neutropenia, 3 cases (Grade 3, 1 case; Grade 4, 2 cases); and thrombocytopenia, 1 case (Grade 4). Complications occurred in 2 cases of radiotherapy: Pneumonitis $(n=1)$ and psychosis $(n=1)$.

The median length of hospitalization was 40 days (range, 24-170 days), 80 days (range, 73-86 days), 153 days (53-227 days), 
Table II. Patient treatment course.

\begin{tabular}{|c|c|c|c|c|c|c|c|c|c|c|}
\hline Case & Age & Sex & Histology & Stage & $\begin{array}{l}\text { Psyhiatric } \\
\text { disorder }\end{array}$ & PS & $\begin{array}{l}\text { Length of } \\
\text { hospital } \\
\text { stay (day) }\end{array}$ & Treatment & Survival & $\begin{array}{c}\text { Survival } \\
\text { time } \\
\text { (months) }\end{array}$ \\
\hline 1 & 65 & $\mathrm{~F}$ & Ad & IA1 & Schizophrenia & 0 & 29 & Surgery & Survival & 61.1 \\
\hline 2 & 61 & $\mathrm{~F}$ & $\mathrm{Ad}$ & IA1 & Schizophrenia & 0 & 24 & Surgery & Survival & 38.7 \\
\hline 3 & 58 & M & $\mathrm{Ad}$ & IA 2 & Schizophrenia & 0 & 24 & Surgery & Survival & 12.8 \\
\hline 4 & 68 & $\mathrm{~F}$ & $\mathrm{Ad}$ & IA 2 & Schizophrenia & 0 & 34 & Surgery & Survival & 85.6 \\
\hline 5 & 74 & $\mathrm{~F}$ & $\mathrm{Ad}$ & IA3 & Mood disorder & 0 & 36 & Surgery & Death & 74.7 \\
\hline 6 & 60 & M & $\mathrm{Ad}$ & IA 3 & Schizophrenia & 0 & 26 & Surgery & Death & 22.5 \\
\hline 7 & 62 & M & $\mathrm{Ad}$ & IA3 & Schizophrenia & 1 & 58 & Surgery & Survival & 13.1 \\
\hline 8 & 55 & M & $\mathrm{Ad}$ & IIIA & Schizophrenia & 0 & 57 & Surgery & Survival & 7.3 \\
\hline 9 & 62 & M & $\mathrm{Ad}$ & IIIA & Schizophrenia & 0 & 189 & Surgery & Survival & 4.4 \\
\hline 10 & 75 & M & $\mathrm{Ad}$ & IIIA & Schizophrenia & 1 & 33 & Best supportive care & Death & 3.9 \\
\hline 11 & 69 & $\mathrm{~F}$ & $\mathrm{Ad}$ & IVc & Schizophrenia & 3 & 20 & Best supportive care & Death & 1.0 \\
\hline 12 & 64 & M & $\mathrm{Sq}$ & IA 2 & Schizophrenia & 0 & 92 & Surgery & Survival & 29.9 \\
\hline 13 & 66 & M & $\mathrm{Sq}$ & IA 2 & Schizophrenia & 0 & 46 & Surgery & Survival & 92.4 \\
\hline 14 & 69 & M & $\mathrm{Sq}$ & IA 3 & Schizophrenia & 0 & 102 & Surgery & Death & 19.5 \\
\hline 15 & 59 & M & $\mathrm{Sq}$ & IIB & Schizophrenia & 0 & 40 & Surgery & Death & 76.7 \\
\hline 16 & 64 & $\mathrm{~F}$ & $\mathrm{Sq}$ & IIIA & Schizophrenia & 0 & 182 & Chemoradiotherapy & Death & 14.2 \\
\hline 17 & 73 & M & $\mathrm{Sq}$ & IIIA & Mood disorder & 2 & 227 & Chemoradiotherapy & Death & 27.5 \\
\hline 18 & 62 & $\mathrm{~F}$ & $\mathrm{Sq}$ & IIIB & Schizophrenia & 0 & 73 & Radiotherapy & Death & 27.7 \\
\hline 19 & 76 & $\mathrm{~F}$ & $\mathrm{Sq}$ & IIIB & Schizophrenia & 2 & 86 & Radiotherapy & Survival & 6.0 \\
\hline 20 & 74 & M & NSCLC-NOS & IIIB & Mood disorder & 0 & 53 & Chemoradiotherapy & Death & 13.0 \\
\hline 21 & 53 & M & SCLC & IIA & Schizophrenia & 0 & 43 & Best supportive care & Death & 2.3 \\
\hline 22 & 61 & $\mathrm{~F}$ & SCLC & IIIA & Mood disorder & 0 & 153 & Chemoradiotherapy & Survival & 64.1 \\
\hline 23 & 73 & $\mathrm{~F}$ & SCLC & IVa & Schizophrenia & 3 & 13 & Best supportive care & Death & 0.8 \\
\hline 24 & 63 & M & SCLC & IVa & Schizophrenia & 1 & 142 & Chemoradiotherapy & Death & 6.6 \\
\hline
\end{tabular}

F, female; M, male; Ad, adenocarcinoma; Sq, squamous cell carcinoma; NOS, not otherwise specified; SCLC, small cell lung carcinoma.

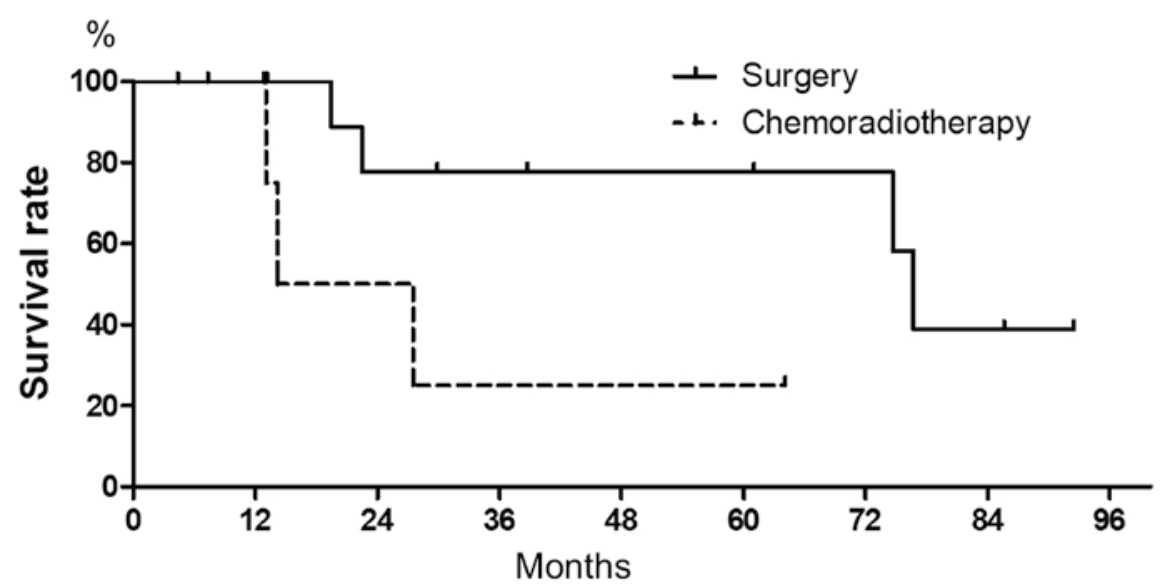

Figure 1. Kaplan-Meier curves for overall survival. Dotted line, overall survival in those who underwent chemoradiotherapy; solid line, overall survival in those who underwent surgery.

and 142 days among those who underwent surgery, radiation therapy, chemoradiotherapy, and chemotherapy alone, respectively (Table II). The prolonged hospitalization was because it was difficult for the patients to admit to our hospital regularly for chemotherapy or radiotherapy due to the lack of caregiver manpower; thus, patients stayed in the hospital until the entire treatment course was completed.

The median survival time was 76.7 months among those who underwent surgery and 14.4 months among those who underwent chemoradiotherapy (Fig. 1). All patients and their 
Table III. Treatment complications.

\begin{tabular}{lll}
\hline Treatment modality & \multicolumn{1}{c}{ Complication } & No. of patients \\
\hline Surgery & Aspiration pneumonia & 1 \\
& Fistulous empyema & 1 \\
& Psychosis & 3 \\
Chemoradiotherapy & Pneumonitis & 2 \\
& Neutropenia & 3 \\
Radiation & Thrombocytopenia & 1 \\
& Aspiration pneumonia & 1 \\
Chemotherapy & Psychosis & 1 \\
\hline
\end{tabular}

families refused adjuvant chemotherapy and further treatment for recurrent lung cancer; thus, the median disease-free survival and progression-free survival could not be evaluated.

\section{Discussion}

The prognosis of patients with lung cancer with schizophrenia is considerably poor (5) because they generally do not receive standard treatment modalities due to various reasons, including the patient wishes, medical comorbidities, psychiatric symptoms, and deficiency of subjective symptoms (8). Patients with psychiatric disorders are difficult to diagnose and treated because they sometimes have poor cooperation making it challenging to obtain consent for diagnosis and treatment. The most important obstacle is psychomotor excitement, which often includes impulsive or poorly controlled activities. For example, positive symptoms including psychomotor excitement are often displayed by patients with acute-phase schizophrenia (9). Our MPU was established for patients who have both physical and severe psychiatric disorders (10). In the MPU, the patients' mental status is assessed daily by psychiatrists, and psychotropic drugs are prescribed to prevent psychomotor excitement.

To our knowledge, this is the first study to describe the profile of patients with lung cancer who have severe psychiatric disorders and who are treated in the MPU. Our study demonstrated the safety profile and tolerable outcome of patients with these concomitant conditions. Invasive examinations and treatment for lung cancer were performed in spite of the patients' psychiatric disorders through cooperation with psychiatrists. There were no complications during or after bronchoscopy and CT-guided biopsy. The frequency and severity of all treatment complications were found to be equivalent to those of lung cancer patients without severe psychiatric disorders (11).

The American College of Chest Physicians and the British Thoracic Society (BTS) recommends that forced expiratory volume in one second (FEV1) and the diffusing capacity for carbon monoxide (DLCO) be measured preoperatively in all patients with lung cancer who are scheduled for surgical resection $(12,13)$. The risk of complications after lung resection has been shown to be inversely related to both FEV1 and DLCO (14). The lung function could not be tested in the patients because they were not able to follow instructions. Tests for arterial blood gas have also been used to evaluate pulmonary function before thoracic surgery to prevent subsequent respiratory complications (15). Therefore, the patients' lung function was assessed according to their arterial blood gas levels in the MPU.

The mental status of 4 patients worsened during treatment. Symptoms of psychosis included delirium and restlessness. Psychosis of three patients appeared after the reduction of psychotropic medications. However, these symptoms were controlled by restorations to original medications and additions of sedatives.

One past study reported that curative surgery can be safely performed in lung cancer patients with schizophrenia, and a high 5-year survival rate can be expected (6). In the current study, we also confirmed that surgery can lead to long-term survival in patients with lung cancer with severe psychiatric disorders. This indicates that surgery should be considered as the primary treatment modality for these patients.

In addition, the median survival time among patients who underwent chemoradiotherapy was as short as that of lung cancer patients without psychiatric disorder (16). The duration of other treatment modalities was longer than that of surgery because of the possibility of posttreatment complications and the necessity of admission until the whole treatment is completed.

There are other issues that need to be considered in the management of patients with lung cancer with psychiatric disorders such as obtaining informed consent and the appropriate follow-up strategy. In the current study, informed consent was obtained from all patients and/or their legal guardian or key family member because it is difficult to evaluate patients' awareness of the disease mortality. Therefore, the legality of a consent form obtained from patients with psychiatric conditions is difficult to determine.

There are some limitations to this study. First, this study was restricted to patients with histologically or cytologically diagnosed lung cancer. The patients' abilities to cooperate for an invasive biopsy showed that they may be more likely to be candidates for cancer treatment. Second, the number of patients in this study was small. Therefore, there were not any statistical analyses. Especially, the number of patients who underwent non-surgical treatment was limited. The effectiveness of radiotherapy and chemotherapy for psychiatric patients is inconclusive. Third, most of the patients were referred to our hospital due to chest radiograph abnormalities, and chest radiograph examinations were performed a few times yearly in the previous hospital. The examinations may improve the outcome of lung cancer patients with severe psychiatric disorder.

In summary, patients with early-stage lung cancer with severe psychiatric disorders who are admitted to the MPU can safely achieve long-term survival. Thus, we concluded that patients with early-stage lung cancer should be treated as ordinary ones. The specific psychiatric disorder should not limit the opportunity of patients for receiving standard treatments for lung cancer.

\section{Acknowledgements}

Not applicable. 


\section{Funding}

No funding was received.

\section{Availability of data and materials}

The datasets used and/or analyzed during the present study are available from the corresponding author on reasonable request.

\section{Authors' contributions}

TTak and TTan conceived and designed the study. TTak, TTan, AI, TY and HK developed the methodology. TTak, TTan, HT and RT acquired the data. TTak, TTan, RT, AI, TY and HK analysed and interpreted the data. TTak, TTan and HK wrote, revised and reviewed the manuscript. TTan and HK supervised the study. All authors read and approved the final manuscript.

\section{Ethics approval and consent to participate}

The Ethical review board committee of Tachikawa Hospital (Tokyo, Japan) approved the present study.

\section{Patient consent for publication}

Not applicable.

\section{Competing interests}

The authors declare that they have no competing interests.

\section{References}

1. Siegel R, Miller KD and Jemal A: Cancer statistics, 2018. CA Cancer J Clin 68: 7-30, 2018.

2. de Leon J and Diaz FJ: A meta-analysis of worldwide studies demonstrates an association between schizophrenia and tobacco smoking behaviors. Schizophr Res 76: 135-157, 2005.

3. Landow L, Szetela B and Know MA: Reducing smoking among psychiatric inpatients: A survey of psychiatrists. Am J Public Health 85: 1169, 1995.
4. Catts VS, Catts SV, O'Toole BI and Frost AD: Cancer incidence in patients with schizophrenia and their first-degree relatives-A meta-analysis. Acta Psychiatr Scand 117: 323-336, 2008.

5. Tran E, Rouillon F, Loze JY, Casadebaig F, Philippe A, Vitry F and Limosin F: Cancer mortality in patients with schizophrenia: An 11-year prospective cohort study. Cancer 115: 3555-3562, 2009.

6. Obuchi T, Okabayashi K, Imakiire T, Yoneda S and Iwasaki A: Outcomes of surgery in lung cancer patients with schizophrenia. Surg Today 44: 855-858, 2014.

7. Miyagawa S, Inoue Y and Higa K: Medical and psychiatric treatment in liaison psychiatric ward of a general hospital. Jap J General Hospital Psychiatry 24: 245-252, 2012 (In Japanese).

8. Mateen FJ, Jatoi A, Lineberry TW, Aranguren D, Creagan ET, Croghan GA, Jett JR, Marks RS, Molina JR and Richardson RL: Do patients with schizophrenia receive state-of-the-art lung cancer therapy? A brief report. Psychooncology 17: 721-725, 2008.

9. Carpenter WT Jr, Strauss JS and Bartko JJ: The diagnosis and understanding of schizophrenia. Part I. Use of signs and symptoms for the identification of schizophrenic patients. Schizophr Bull 37-49, 1974.

10. Fava GA, Wise TN, Molnar G and Zielezny M: The medical-psychiatric unit: A novel psychosomatic approach. Psychother Psychosom 43: 194-201, 1985.

11. Myrdal G, Gustafsson G, Lambe M, Horte LG and Stahle E: Outcome after lung cancer surgery. Factors predicting early mortality and major morbidity. Eur J Cardiothorac Surg 20: 694-699, 2001

12. British Thoracic Society; Society of Cardiothoracic Surgeons of Great Britain and Ireland Working Party: BTS guidelines: Guidelines on the selection of patients with lung cancer for surgery. Thorax 56: 89-108, 2001.

13. Brunelli A, Kim AW, Berger KI and Addrizzo-Harris DJ: Physiologic evaluation of the patient with lung cancer being considered for resectional surgery: Diagnosis and management of lung cancer, 3rd ed: American College of Chest Physicians evidence-based clinical practice guidelines. Chest 143 (Suppl 5): e166S-e190S, 2013.

14. Ferguson MK and Vigneswaran WT: Diffusing capacity predicts morbidity after lung resection in patients without obstructive lung disease. Ann Thorac Surg 85: 1158-1164; discussion 1164-1165, 2008.

15. Tisi GM: Preoperative evaluation of pulmonary function. Validity, indications, and benefits. Am Rev Respir Dis 119: 293-310, 1979.

16. Yoon SM, Shaikh T and Hallman M: Therapeutic management options for stage III non-small cell lung cancer. World J Clin Oncol 8: 1-20, 2017. 